\title{
Livres vivants
}

\section{Christian Jacob}

\section{(2) OpenEdition}

\author{
Journals
}

Édition électronique

URL : https://journals.openedition.org/rbnu/2919

DOI : $10.4000 /$ rbnu.2919

ISSN : 2679-6104

\section{Éditeur}

Bibliothèque nationale et universitaire de Strasbourg

\section{Édition imprimée}

Date de publication : 1 mai 2015

Pagination : 8-17

ISBN : 978-2-85923-058-6

ISSN : 2109-2761

\section{Référence électronique}

Christian Jacob, «Livres vivants », La Revue de la BNU [En ligne], 11 | 2015, mis en ligne le 01 novembre 2020, consulté le 18 juin 2021. URL : http://journals.openedition.org/rbnu/2919 ; DOI : https://doi.org/ $10.4000 /$ rbnu. 2919

\section{(c) (i) (3) (2)}

La Revue de la BNU est mise à disposition selon les termes de la Licence Creative Commons Attribution - Pas d'Utilisation Commerciale - Partage dans les Mêmes Conditions 4.0 International. 

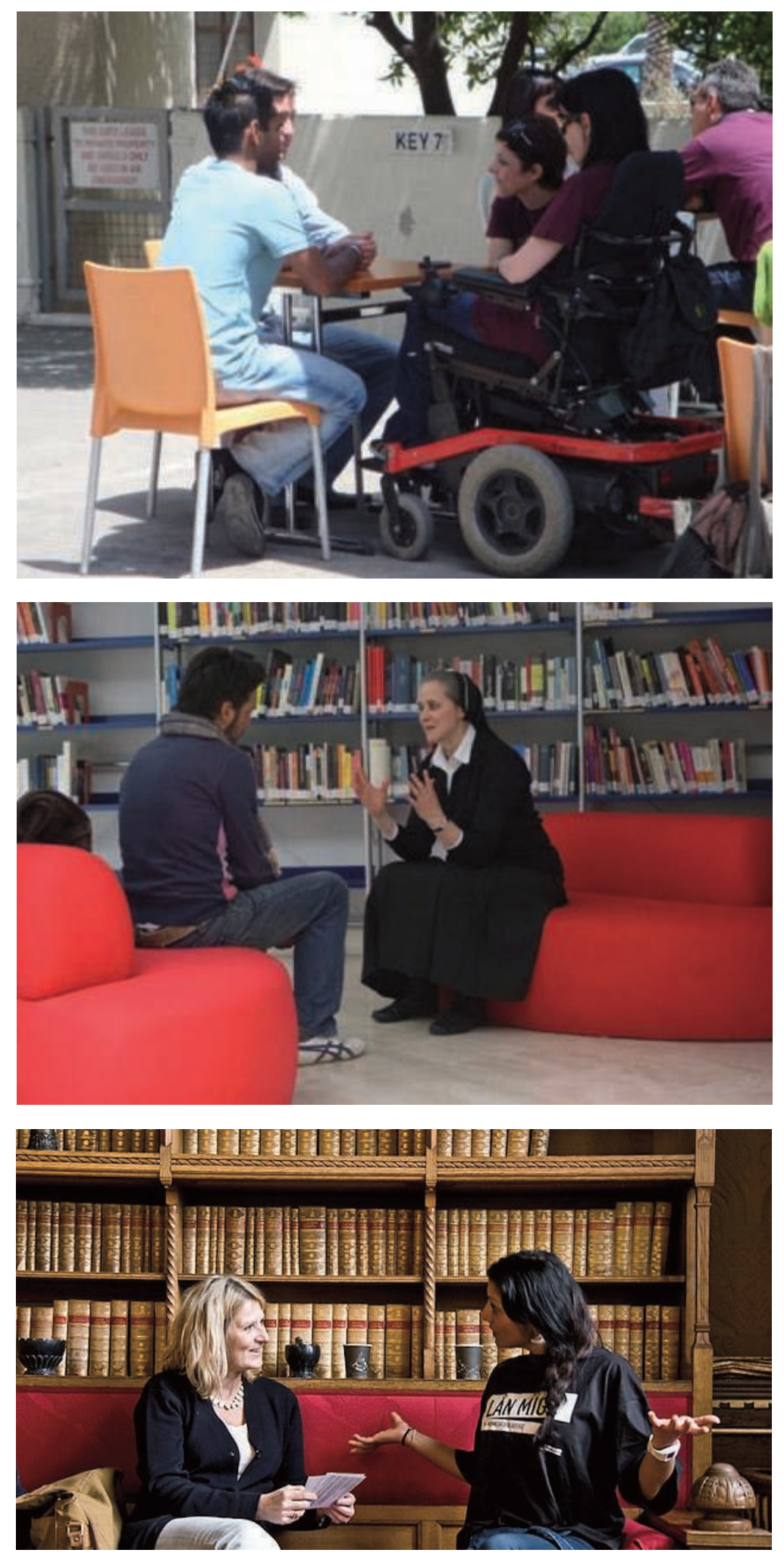

(de haut en bas) :

Une personne handicapée et ses «lecteurs» (Limassol, Chypre, 2009)

Une religieuse et son «lecteur" (Terni, Italie, 2010)

Une rappeuse et sa «lectrice» (Copenhague, 2010) 


\section{Livres vivants}

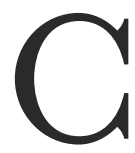

'est un article du Monde, en date du 29 décembre 2010, qui a attiré mon attention sur l'une des innovations les plus marquantes de ces dernières années dans le domaine des bibliothèques, du livre et de la lecture. Bien au-delà du web, même du web 2.o, il s'agit d'une technologie inédite de la communication et de la transmission des savoirs et des pensées. Ce dispositif expérimental, tout juste devenu public, répond à un défi majeur, car il a réussi à transcender la critique platonicienne de l'écrit et du livre, jugés décevants car ils ne font que répéter la même chose à tous leurs lecteurs, et ne répondent pas à leurs questions dans ce qu'elles ont de personnel et d'imprévisible. L'e-book, dans la diversité de ses formats, entre e-pub, mobi et pdf, est d'ores et déjà dépassé. Les Kindle, Ipads et autres liseuses redeviennent les ardoises magiques de notre enfance.

Qui est à l'origine de ce livre de la prochaine génération ? Ce n'est ni Apple ni Google, ni Amazon ni Microsoft. Ce n'est pas le MIT ni une start-up de la Silicon Valley. Ce sont des organisations non-gouvernementales, relayées par des bibliothécaires, qui ont travaillé longuement pour imaginer, matérialiser et tester ce qui promet de révolutionner le monde de la lecture contemporaine et de donner envie de lire même à ceux qui sont les plus réfractaires au monde de l'écrit. Ces utopistes ont en effet réussi à concevoir un nouveau modèle de livre, basé sur un concept révolutionnaire, un livre intelligent, fluide, évolutif, autonome, personnalisable, en métamorphose perpétuelle. Ce nouveau livre fonde aussi un nouveau mode de lecture, allant bien au-delà du parcours silencieux ou murmuré de notre regard sur la surface d'une page, manuscrite ou imprimée. Ce magnifique dispositif offre au lecteur une expérience inédite, complexe, multisensorielle et véritablement interactive.
Comme ceux que nous connaissons aujourd'hui, ces livres d'un nouveau genre ont un titre et ils ont des histoires à raconter, des savoirs à transmettre. Comme les livres de nos bibliothèques, on peut les réserver et les emprunter pour un temps, même si la consultation, pour le moment, doit se faire en bibliothèque ou dans d'autres lieux où le dispositif est provisoirement installé. Ce sont en effet principalement des bibliothèques qui permettent de tester cette nouvelle technologie, et on ne trouve pas encore ces livres de nouvelle génération dans nos librairies réelles ou virtuelles. Ces livres s'empruntent, se partagent, mais ne s'achètent pas. On ne peut pas non plus les télécharger, que ce soit sur des sites de streaming, des plateformes légales ou les réseaux " peer to peer ". Ce sont des prototypes, des livres d'une nouvelle génération tant ils renouvellent les pratiques de la lecture, le dialogue du lecteur avec ce qu'il lit. Ils permettent une forme d'interactivité inédite, un processus d'où le lecteur et le livre lui-même ne sortiront pas tout à fait indemnes, ou en tout cas pas semblables à ce qu'ils étaient avant de se rencontrer.

Les premières expérimentations remontent à l'an 2000, au Danemark, où le concept est né dans des milieux alternatifs et des organisations non-gouvernementales. Le succès fut immédiat, et on importa le dispositif en Hongrie et en Norvège. Ce nouveau genre de livre reçut alors un soutien officiel du Conseil européen, et commença à être diffusé largement dans les bibliothèques publiques, les salons du livre et les lieux d'enseignement.

En 2011, après une première expérimentation couronnée de succès, la bibliothèque publique de Toronto, dans la province de l'Ontario, au Canada, créa le premier département dédié à ce nouveau support, à côté des livres imprimés, des e-books, des DVD et des magazines. 
Toronto représente le plus grand système de bibliothèques publiques de l'Amérique du Nord, avec quatrevingt-dix-neuf succursales dans l'agglomération. Elle met à disposition de ses lecteurs onze millions de documents, dans cent langues différentes. Parmi ses collections, on trouve désormais ces livres inédits que je voudrais vous faire découvrir dans ces quelques lignes.

Peut-être avez-vous déjà reconnu les livres humains, ces hommes et ces femmes-livres dont la conception et la mise au point sont à mettre au crédit de l'organisation " The Human Library ".

Permettez-moi de citer la description qu'en donne cette ONG : « La bibliothèque vivante fonctionne exactement de la même façon qu'une bibliothèque classique. Les lecteurs viennent y emprunter un livre pour une durée limitée; quand ils ont fini de le lire, ils le ramènent à la bibliothèque et peuvent en emprunter un autre, s'ils le désirent. Mais il y a une petite différence : les livres de la bibliothèque vivante sont des êtres humains. Entre eux et leurs lecteurs, un dialogue va s'installer. Les livres sont des personnes qui représentent des groupes fréquemment confrontés à des préjugés et à des stéréotypes, souvent victimes de discrimination et/ou d'exclusion sociale. Le lecteur peut être n'importe qui désirant dialoguer avec son propre préjugé ou stéréotype et étant prêt à consacrer une heure à cette expérience. Dans la bibliothèque vivante, les livres ont la parole : ils sont en mesure de répondre aux questions du lecteur, voire de lui poser des questions et ainsi d'apprendre, eux aussi ".

Lors des premiers tests menés à la Bibliothèque publique de Toronto, deux cents lecteurs ont été sélectionnés, pour une collection de soixante livres humains. Chaque lecteur pouvait emprunter un livre humain pour une durée de 30 minutes et converser avec lui, avant de le restituer au service du prêt pour qu'un autre lecteur puisse l'emprunter à son tour. Anne Pélouas, correspondante du Monde à Montréal, nous indique qu'un moine bouddhiste tibétain, réfugié au Canada, comptait parmi les titres les plus demandés, de même qu'un ancien membre d'un gang devenu guitariste dans un groupe à succès.

C’est sur cette nouvelle pratique lettrée, qui a été expérimentée à Paris, à la Cité internationale universitaire et au Collège des Bernardins, notamment par l'artiste Fanny de Chaillé, que je voudrais poursuivre ma réflexion. Qu'est-ce qu'un livre, qu'est-ce qu'un lecteur, qu'est-ce que lire?

La lecture est une rencontre, la rencontre entre un lecteur et un texte. Cette rencontre peut être aléatoire ou élective, répondre à la logique d'un projet intellectuel ou spirituel comme à l'impulsion d'une curiosité, s'inscrire dans une activité professionnelle ou dans le loisir, dans l'apprentissage ou dans une quête, ou encore dans un désir d'horizons, un désir d'évasion. Comment et pourquoi choisissons-nous de lire un livre ? Il y aurait sans doute un parallèle à établir entre le rayonnage de la bibliothèque, la table de présentation de la librairie, l'écran des bookstores virtuels, qui se prêtent à la recherche raisonnée comme au libre vagabondage. Qu'est-ce qui nous conduit à choisir un livre ? Est-ce un nom d'auteur, un titre, l'estampille d'une collection ou encore le design d'une couverture ? Comme l'indique non sans malice le projet Human Library, "la couverture ne fait pas le livre !" Il faut aller au-delà des apparences, aller au-delà d'un visage ou d'une silhouette pour entrer dans l'univers d'un autre. Choisir un livre est affirmer une attente, un désir, une confiance : on fait le premier pas d'un voyage dont on ignore la destination finale. La lecture est une rencontre de l'autre, une parenthèse où se redessinent la distance et la proximité, l'identité et l'altérité. Lire, c'est parler, penser, sentir, imaginer, se souvenir du point de vue d'un autre, sans que jamais la différence entre l'un et l'autre ne s'abolisse tout à fait.

Même si les livres humains ne sont pas à vendre, on trouve cependant parmi eux des best-sellers : ce sont les livres les plus demandés par les lecteurs, qui ne restent jamais très longtemps sur le rayonnage et qui sont en permanence empruntés. Qu'est-ce qui fait le succès d'un livre humain ? Le bouche à oreille, sans aucun doute, et la prescription d'une communauté de lecteurs, des voix qui y font autorité. Dans la belle expérience de l'organisation des bibliothèques humaines, on a remarqué que les best-sellers étaient les livres s'inscrivant dans l'actualité de leur milieu de réception, apportant une expérience, un point de vue particuliers à des questions cruciales, la violence, le droit, la cité, le racisme, la pauvreté, le handicap, le combat politique. Ce qui dans le best-seller touche à l'universalité est précisément ce qui en lui est le plus singulier, le plus personnel, ici et maintenant.

À la bibliothèque de Toronto, la temporalité de la lecture d'un livre humain est singulièrement condensée : trente minutes, pas une de plus, pour ne pas fatiguer le livre, pour satisfaire d'autres lecteurs. Pour ne pas fatiguer le livre, car il doit pouvoir se reposer entre deux lecteurs, avant de parler à nouveau à d'autres oreilles et de s'ouvrir à d'autres regards. Une lecture continue trop longue peut rendre le livre muet, de même que Borges rêve à des livres imprimés dont les pages s'effaceraient progressivement comme des lettres sur le sable, usées 
ESSAIS DE M. DE MONTA. \&. auffi peu d'enchantement, ayant ouy faire yét conte à vn fié compagnon, d'vne defaillace extraordinaire, en quoy il eftoit tombe fur le point, quil en auoit le moins de befoin, fe trouuant en pareille occafion; ! horreur de ce conteluy uint,if fu-

Ef dela irfors

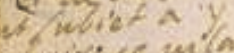
chollice wim

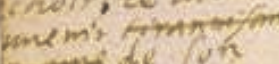
couruenisht te onrmardirat firk: ant. PA Grund

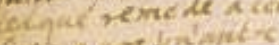

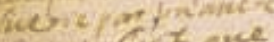
iceric, eisf quce weisant ligyind ine

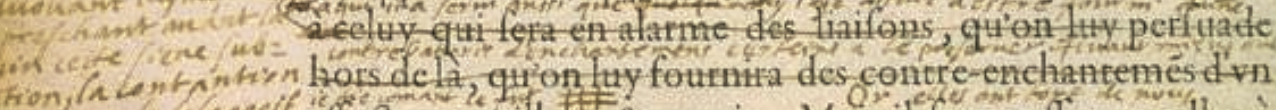

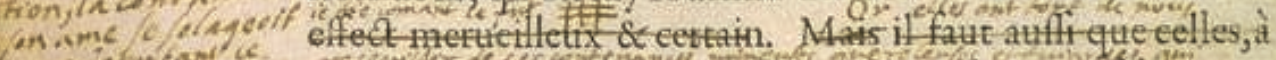

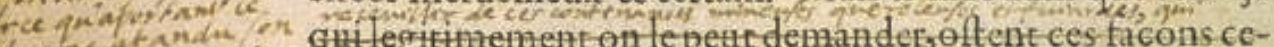

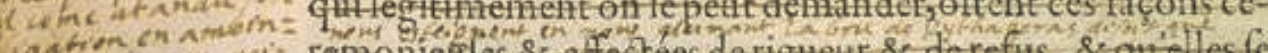
iection on am iel:

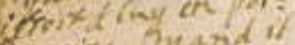

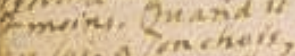

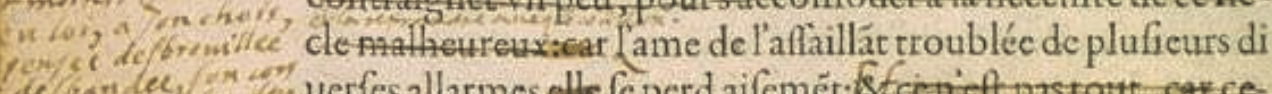

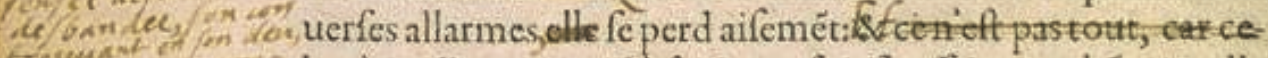

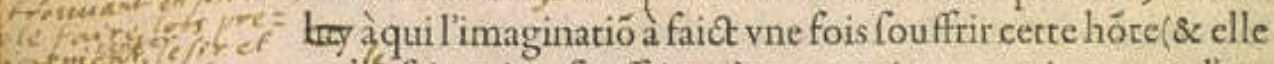
tormenty $7 x$

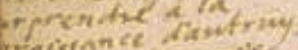

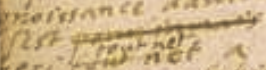

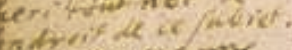
inen nowe me

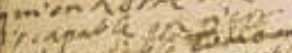
ne 1 a fait guiere fouffrir quiaux premieres accointances, dautant qu'elles font plus ardantes \& afpres; \&caufi qu'en certe premiere connoillance qu'ondonne defoy, on crainc beaucoup plus de faillir) ayane mal commencé, il entre en ffyranSin costrinitiver de fieure \& defpit de cet accident, que cete frayetrienaugthétéc luy redouble àtotrtestes occafions fuiuanres: \& fatis - cs mmriez ont quelque contre-mineonn'en viétpasaifément àbout.T $\mathrm{d}$ à$\rightarrow$ enturenture par cet effect de limagination, laiffe icy les ef-

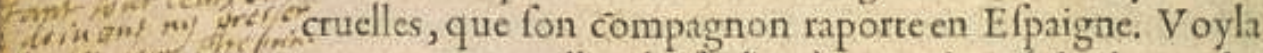
tas en telles chofes lon à accouftumé de demander

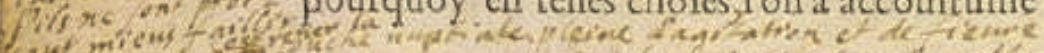

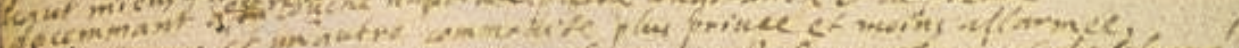

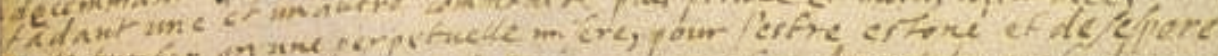

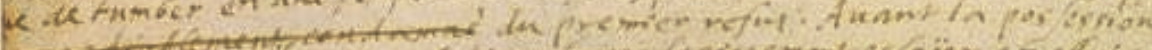

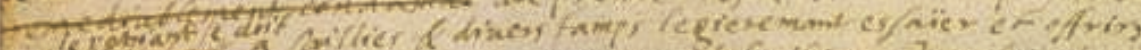

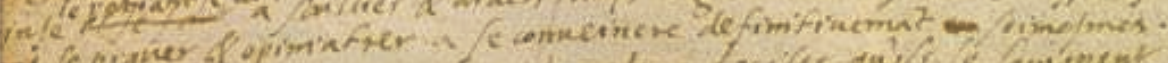

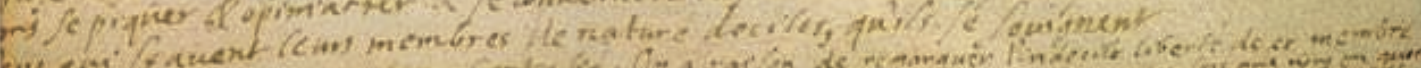

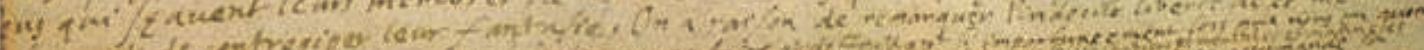

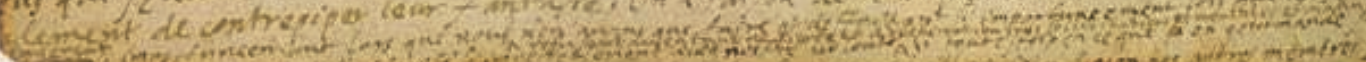




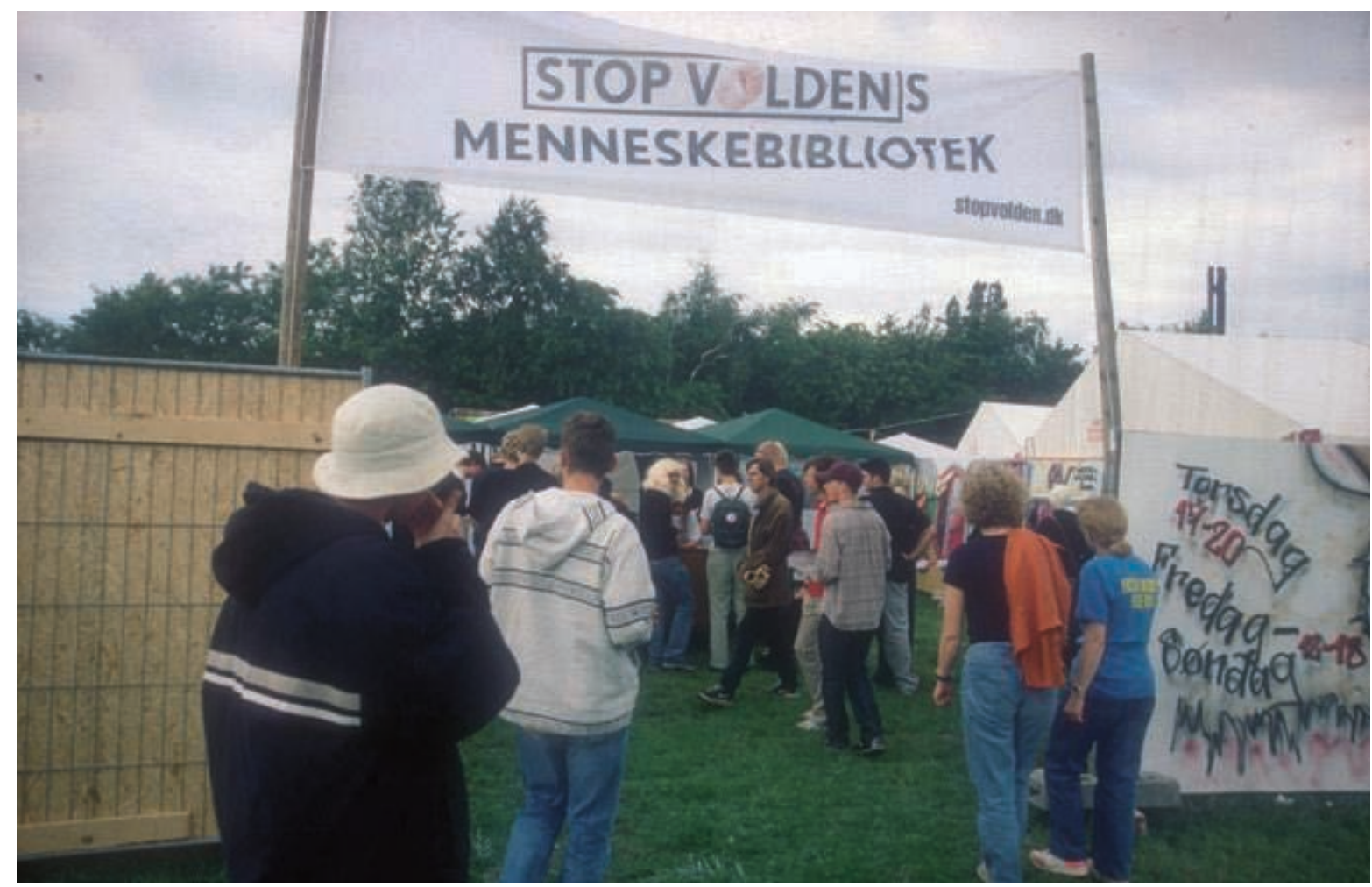

Entrée principale de la première bibliothèque vivante,

tenue lors du festival Roskilde en 2000. La bannière dit : Bibliothèque vivante "Stop à la violence». 
par les regards comme une plage balayée par les vents. Trente minutes seulement, donc, pour ne pas faire attendre trop longtemps les autres lecteurs, car tout livre d'une bibliothèque passe de main en main, d'un lecteur à l'autre. Le lecteur est un maillon dans une chaîne d'attente et de désir et il me plaît d'imaginer que tout livre d'une bibliothèque se prête à ce que nous appellerions aujourd'hui une "lecture enrichie ", car entre les pages et les lignes, il garde trace des regards, des imaginaires, des intelligences qui l'ont lu avant moi, et qui ajoutent des couches sédimentées d'interprétation et de rêve. De ce point de vue, les livres des bibliothèques sont des palimpsestes.

Il est difficile d'établir une typologie des lecteurs qui fréquentent les bibliothèques. Pour un observateur fin, sans doute, chaque lecteur serait une catégorie en soi, et on serait tenté d'adopter une taxonomie aussi fine que celle des naturalistes travaillant sur les insectes ou sur les plantes. On pourrait aussi déployer la plus complète des analyses ethnographiques et éthologiques sur la communauté des lecteurs et des conservateurs de bibliothèques, en nous attachant aux rituels sociaux, aux codes de comportement, aux gestes et aux instruments, aux technique du corps, aux classes d'âge et à la division des sexes. Je me contenterai ici de dresser le tableau à grands traits, et de répartir les lecteurs de nos bibliothèques en deux grandes catégories, les lecteurs intensifs et les lecteurs extensifs. Les premiers choisissent un livre et cheminent mot à mot, lisent pas à pas, creusent sous la surface du texte et regardent même entre les lignes, tant ils cherchent à creuser la stratigraphie du sens, à déployer le réseau des liens qui rattachent tout texte à une bibliothèque synchronique et diachronique. Les lecteurs extensifs, quant à eux, passent d'un livre à l'autre, font jouer les livres entre eux par de multiples liens de correspondances, de complémentarité, de présupposition. Ce sont des voyageurs, des nomades, et les meilleurs lecteurs sont ceux qui partent à l'aventure sans préjuger de leur destination. Ce qui compte pour eux est l'itinéraire de livre en livre autant que l'arrêt sur la page. Cet itinéraire peut traverser un paysage continu ou provoquer un dépaysement brutal : en ouvrant un livre, le lecteur peut choisir de poursuivre un voyage et de changer d'univers ou de rebrousser chemin.

Les bibliothèques humaines croisent les deux types d'expériences. Elles invitent à un face à face intensif avec un livre particulier dont on tentera d'explorer l'univers, de capter le savoir et d'écouter la musique propres. Mais l'expérience fait sens également par le parcours auquel elle invite d'un livre à l'autre, par les échos et les contrastes, la continuité ou les ellipses que le lecteur peut ressentir. Un lecteur méthodique peut ainsi emprunter plusieurs livres humains pendant une même journée.

La lecture se déroule selon une temporalité linéaire non réversible, même si le lecteur peut contrôler le rythme : arrêter, ralentir, accélérer, aller en arrière, sauter des pages... Mais toute lecture a une fin, qu'il s'agisse d'un livre manuscrit, imprimé, numérique ou d'un livre humain. Comment prend-on congé d'un livre ? La séparation fait partie de l'expérience de la bibliothèque. Et tout lecteur doit dire au revoir, et parfois adieu, au livre qu'il restitue. Le lecteur n'a qu'un droit d'usage temporaire, un droit de passage, le livre ne fait que passer entre ses mains et sous ses yeux. Si la lecture est une expérience inscrite dans un temps plus ou moins circonscrit, elle laisse cependant une trace durable dans la mémoire du lecteur : au fond, que retient-on d'un livre ? Un ensemble d'impressions sur les tablettes de la mémoire, des impressions qui s'échelonnent de l'affectif à l'analytique, du halo imaginaire aux idées et aux informations mémorisées. Cette trace peut être durable ou évanescente, elle peut accompagner le lecteur sa vie durant ou s'effacer peu à peu.

Tout lecteur doit affronter ce dilemme : que retenir de ses parcours dans un livre, dans une bibliothèque? Comment les archiver et les préserver dans sa mémoire? La question est particulièrement aiguë pour les livres que l'on lit ou emprunte dans une bibliothèque. Certains lecteurs acceptent de vivre pleinement le temps de la lecture sans songer à son au-delà. D'autres en revanche essaient de renforcer leur mémoire vive par une mémoire externe et matérialisée : notes de lecture, photocopies, voire numérisation partielle, lorsque c'est possible. On tente de capter l'essentiel d'une réflexion, les jalons d'un récit, les articulations d'un savoir, ou simplement des mots-clés qui permettront plus tard de réveiller la mémoire, ou encore quelques harmoniques subjectives qui feront renaître la musique du texte lu. Lire, c'est lutter contre la perte et l'oubli, mais aussi pour la possibilité du sens et de la mémoire, qui reposent sur la sélection, sur des grilles de tamisage et de filtrage qui retiendront des objets de langage et de savoir particuliers, en laissant filer la poussière du texte. Dans la bibliothèque humaine, le lecteur peut enregistrer le livre qui parle, prendre des notes sténographiques, le filmer, le photographier, le dessiner, autant de traces analogiques qui capteront des pans singuliers de l'expérience vécue sans jamais en restituer la totalité. 
Erllärung des britischen Außenministers, Michael Stewart, anläßlich

der Unterzeichnung des Vertrags tiber die Nichtverbreitung von Kernwaffen durch die Bundesrepublik Deutschland am 28. November 1969

Betrifft: Bundesrcpublik und NV-Vertrag - Sicherheit der Bundesrepublik - Artikel 53 und 107 der UN-Charta - Gewaltverzicht Beistandspflicht der NATO und WEU.

Sobald ein Vertrag zur Unterzeichnung freigegeben ist und die erste Unterschriftszeremonie stattgefunden hat, entspricht es normalerweise nicht unsere Praxis, yeitere Zeremonien für spätere Unterzeichnerstaaten abzuhalten. Aber dies ist eime ganz besondere Gelegenheit, denn es ist nicht übertrieben, wenn man sagt, daß die gesamte Zukunft dieses Nichtverbreitungsvertrags von der Haltung der Bundesrepublik abhing. Dies mag paradox erscheinen angesichts der Tatsache, daß die Bundesrepublik bereits vor vielen Jahren feierlich auf die Herstellung von Kernwaffen verzichtet hat, aber die heutige Welt ist voller Paradoxe. Wir alle, Eure Exzellenz, kennen die Umstände, die die Unterzeichnung durch Ihr Land so bedeutsam gemacht haben, und wir alle verstehen die Uberlegungen, die Ihrer Regierung die Entscheidung zur Unterzeichnung so schwer gemacht haben.

In den letzten Tagen haben wir die gute Nachricht erhalten, daß die Vereinigten Staaten und die Sowjetunion den Vertrag ratifizieren. Wir sind zuversichtlich, daß diesen Ratifizierungen und der Unterzeichnung durch Deutschland bald weitere Unterschriften und Ratifizierungen folgen werden, und wir hoffen, daß der Vertrag infolgedessen in Kraft treten wird, bevor viele weitere Monate vergehen, und daß er den Weg für weitere Maßnahmen der Rüstungskontrolle und der Abrüstung ebnen wird.

In diesem Zusammenhang möchte ich nun die folgende formelle Erklarung abgeben:

Artikel 53 und 107 der Charta der Vereinten Nationen verleihen kein Recht zu einseitiger gewaltsamer Intervention in der Bundesrepublik Deutschland. Die Regierung Ihrer Majestät sowie die Regierungen Frankreichs und der Vereinigten Staaten von Amerika haben formlich erklärt, daß sie die in Artikel 2 der Charta niedergelegten Grundsätze in thren Beziehungen zur Bundesrepublik befolgen werden. Die anderen Unterzeichner des Nordatiantikvertrags haben sich dieser Brkiarung angeschlossen. Darüber hinaus ist die Bundesrepublik als vollwertige und gleichberechtigte Vertragspartei des Nordathantikvertrags selbstverstindition durch diesen Vertrag geschutz. Nach Artikel V des Vertrags würde ein bewaffneter Angriff gegen die Bundesrepublik Deutschland wie ein Angriff gegen alle Vertragsparteien behandelt werden, die in Ausübung des Rechts der individuellen oder kollektiven Selbstverteidigung Beistand leisten würden, indem sie unverzüglich geeignete $\mathrm{Maßnahmen}$ im Einklang mit dem Vertrag treffen. Gleichermaßen würden nach Artikel V des Brüsseler Vertrags bei einem bewaffneten Angriff in Europa auf eine der Vertragsparteien die anderen Vertragsparteien alle in ihrer Macht stehende militärische und sonstige Hilfe und Unterstuitzung leisten.

Q u ell e: London Press Seroice, 7618, 28. November 1969; deutsch z. T. nach: Bulletin des Presse- und Informationsamtes der Bundesregierung, Nr. 145, 29. November 1969.

\section{Erklärung des französischen Außenministeriums vom 28. November 1969 zur \\ Unterzeichnung des Vertrags über die Nichtverbreitung von Kernwaffen durch die Bundesrepublik Deutschland}

Betriff: Artikel 53 und 107 der UN-Charta.

Wir schließen uns keiner Interpretation der Artikel 53 und 107 der Charta an, derzufolge diese Artikel bestimmten Lăndern das Recht zu einseitiger gewaltsamer Intervention in der Bundesrepublik Deutschland verleihen wïrden.

Quolle: Bulletin des Presse- und Informationsamtes der Bundesregierung, 29, 11. 1969.

Europa-Archiv, Folge 1 / 1970

D 23

Lecture intensive, lutte contre la perte et l'oubli ou livre rendu muet car " usé par les regards " ?

Page d'un ouvrage de la Bibliothèque nationale et universitaire de Strasbourg annoté par ses lecteurs (coll. BNU) 
Il faudrait réfléchir aux métaphores économiques qui structurent certains imaginaires de la lecture : celui de la capitalisation, de l'accumulation, de la thésaurisation en particulier, où l'on est hanté par le risque de la perte et de l'oubli, où l'on fait fructifier ses lectures en accumulant les notes, les photocopies, les fiches, les traces analogiques ou numériques qui préservent de l'oubli des fragments de textes, qui les réorganisent selon des rationalités nouvelles. Certains lecteurs acceptent d'oublier. D’autres sont obsédés par la perte de la totalité. Ils rêvent de devenir eux-mêmes le livre.

Je pense en particulier à la topique du lettré pauvre dans la tradition chinoise, analysée par Jean-Pierre Drège dans son beau livre sur l'histoire des bibliothèques en Chine ancienne :

"La famille de Wang Chong (27-vers 97) était pauvre et n'avait pas de livres. Aussi se promenait-il souvent dans les boutiques du marché de Luoyang pour y regarder les livres qui y étaient en vente. Ce qu'il avait vu une fois, il pouvait le réciter par cœur ; c'est pourquoi il connaissait parfaitement les propos des divers courants et des cent écoles ".

Ou encore à propos de Xun Yue (148-209) : " À l'âge de douze ans, il pouvait expliquer le Chunqiu. Sa famille était pauvre et n'avait pas de livres. Chaque fois qu'il allait chez quelqu'un et qu'il y regardait des livres (tablettes...), il lui suffisait de les voir une fois pour qu'il puisse la plupart du temps les réciter et les noter ".

Ces lecteurs s'approprient la totalité des textes qu'ils balayent du regard. Ils deviennent des livres humains. La seule solution pour eux est de devenir le livre, de l'incorporer et de s'identifier à lui, de l'écrire sur les tablettes de leur mémoire.

Mais revenons aux bibliothèques humaines et à leur service du prêt. La lecture peut être individuelle ou collective et partagée. Elle devient alors une activité sociale, où différentes écoutes se croisent pour partager un texte lu. Si un livre humain peut s'adresser à son lecteur en tête-à-tête, il peut aussi s'offrir à un auditoire plus large. L'historien de la culture écrite se rappellera les multiples formes sociales de la lecture oralisée, voire théâtralisée, sa place dans le quotidien comme dans le festif, dans le profane comme dans le sacré : lecture des textes révélés dans les lieux de la prière, lecture des contes dans les veillées familiales, lecture des classiques dans les écoles, lecture d'une conférence dans un amphithéâtre de la Bibliothèque nationale de France.
Le livre humain est une voix et un corps autant qu'un texte. Les trois composantes sont indissociablement liées, et contribuent à la production d'un sens global, reposant sur les intonations et les rythmes, sur les gestes et les postures comme sur la langue. La compréhension du texte n'est possible que dans la maîtrise des codes mélodiques et chorégraphiques qui en accompagnent la diction. En Grèce ancienne, la lecture oralisée était prédominante, que l'on lise un texte pour soi-même ou pour un cercle d'auditeurs. C'est la voix qui construisait les phrases et séparait les mots, qui marquait la ponctuation et les accents dans le fil continu d'une écriture qui ne présentait aucun trait de structuration visuelle, sinon la superposition des lignes en colonnes, sur le ruban du papyrus. C'est en lisant à haute voix que l'on rendait un texte intelligible, pour soi-même comme pour les autres. Mais ce n'est pas tout. La lecture à haute voix impliquait aussi l'interprétation vocale du texte selon des codes adaptés à chaque genre littéraire, à chaque registre de discours, qu'il s'agisse d'un texte en prose, d'un poème lyrique ou d'un chant d'épopée, d'une comédie ou d'une tragédie. Cette interprétation reposait aussi bien sur le ton de la voix que sur la ligne mélodique et différents registres expressifs qui contribuaient à l'intelligibilité même du texte. Une telle pratique de lecture supposait la maîtrise de différents codes littéraires et culturels, appris dans une tradition de performances orales ancrées dans des cadres sociaux et rituels particuliers. Se tromper dans les codes de la vocalisation comme au niveau plus élémentaire de la transformation de la séquence continue des lettres en phrases découpées en mots trahissait immédiatement l'incompétence et l'ignorance du lecteur en l'exposant aux sarcasmes de ses auditeurs. À un moment donné de la transmission de la littérature grecque, depuis les performances du monde des cités vers les bibliothèques des royaumes hellénistiques, les œuvres ont perdu leurs codes d'interprétation, les textes ont été dépouillés des clés de leur vocalisation et de leur expressivité. Il revint aux bibliothécaires alexandrins de superposer à certains textes, en particulier ceux de la poésie lyrique, des signes conventionnels, comme une méta-écriture, qui pourraient aider à réinventer leur lecture à haute voix, à rétablir leur musicalité : ces signes étaient la ponctuation, les accents mélodiques, ainsi que le découpage des poèmes en strophes faisant ressortir visuellement les unités rythmiques.

Avec les livres humains également, le texte est indissociable d'une voix, le sens est tissé dans les mots et les sons, dans tous les registres de l'expressivité des gestes 
et du visage. Le texte y est mis en voix et en corps, les silences et les intonations, les sourires comme les regards enrichissent les mots des mille subtilités de la communication non verbale, dans le face-à-face du livre et de son lecteur. Celui-ci doit savoir lire entre les mots, et l'essentiel du texte se laisse saisir dans le corps qui le porte.

Poursuivons notre réflexion sur les enjeux de ces bibliothèques humaines. L'un des traits fondamentaux des cultures du livre est que la reproduction écrite des textes permet leur diffusion, leur multiplication, sans qu'ils perdent leur sens, leur pouvoir, leur efficacité. Étonnant processus que celui qui conduit à reproduire un texte en de multiples exemplaires, manuscrits ou imprimés, ou encore fichiers numériques pouvant être téléchargés par un nombre indéterminé d'utilisateurs. On admet que le texte reste identique d'un exemplaire à l'autre, qu'il conserve ses effets, sa forme et son sens, et qu'il pourra livrer le même enseignement, le même savoir à tous ses lecteurs, qu'ils se comptent sur les doigts d'une main ou qu'ils soient des centaines de milliers. La reproduction des textes, manuscrite ou imprimée, leur inscription sur un support matériel qui peut faire l'objet d'une reproduction centralisée ou centrifuge, sont parmi les conditions nécessaires pour faire circuler un savoir, une spiritualité, une littérature dans une société donnée.

Or chaque livre humain est une pièce unique, non reproductible. C'est un objet singulier, qui fait de la lecture une expérience intense, ancrée dans le temps et le lieu d'une rencontre. Les collections des bibliothèques humaines sont composées de livres uniques, qui ont été écrits au cours d'un long processus, d'un temps de vie, d'une trajectoire à nulle autre pareille. Le lecteur ne peut partager sa lecture que dans le cercle minuscule de ceux qui ont eu accès à ce livre, dans un lieu et un temps déterminés. Ce mode de lecture est extraordinairement intime et singulier. Mais même dans ce cercle, l'expérience que lui a offerte le livre humain est d'une singularité absolue : car le texte du livre est fluide, évolutif, en métamorphose perpétuelle, et il change d'un lecteur à l'autre.

Les bibliothèques humaines de ce $21^{\mathrm{e}}$ siècle semblent s'inscrire dans une longue généalogie. Déjà, au $1^{\mathrm{er}}$ siècle de notre ère, Sénèque (Lettres, 27, 5-8) évoquait un contemporain, Calvisius Sabinus, qui avait acheté au prix fort des esclaves entraînés à être des livres vivants : chacun avait mémorisé un classique - Homère, Hésiode ou les poètes lyriques. Pour se constituer une bibliothèque, il fallait donc commander des esclaves-livres auprès de fournisseurs spécialisés. Ces esclaves-livres ne connaissaient qu'un texte, et peut-être les appelait-on par le titre de ce texte, "Iliade ", "Odyssée ", "Théogonie ", ou par son auteur. À la différence des livres sur rouleaux de papyrus, ces livres vivants n'exigeaient pas l'exercice pénible du déchiffrement visuel et de l'interprétation vocale. Ces livres se lisaient d'eux-mêmes à haute voix. Comment ce Romain utilisait-il sa bibliothèque humaine ? Ces livres-esclaves fournissaient à leur maître sans mémoire les citations appropriées lors des conversations de banquet. Ils étaient pour ainsi dire des bases de données vivantes où l'on pouvait naviguer librement et mener des recherches en texte intégral, selon des algorithmes culturels sophistiqués, régis par des critères de pertinence de manière à s'adapter à tout moment d'une interaction sociale. Comme dans Fahrenheit 451 de Ray Bradbury, les esclaves lettrés de Sénèque, ces hommeslivres à défaut d'être libres, sont porteurs d'un texte fixe, d'une œuvre littéraire associée à un nom d'auteur, régie par l'exigence de la littéralité.

La littéralité est aussi régulée par des normes culturelles particulières, qui définiront, pour chaque catégorie de textes, la marge de variation, de changement tolérable dans la reproduction pour que son sens et sa forme ne soient pas considérés comme dénaturés. Ces normes définiront le degré de stabilité des textes dans le processus de reproduction : littéralité stricte, paraphrase, résumé, traduction, transcription, mise à jour, réécriture ; ces différents degrés d'écart peuvent être acceptables ou non, préserver le sens originel d'un texte ou l'adapter à de nouvelles pratiques de lecture, à de nouveaux horizons de réception. Il ne s'agit pas seulement de technique de production des livres, mais des formes d'autorité sociale, politique et religieuse qui régulent leur circulation.

Dans le cas des livres humains, le texte change à chaque lecture. Le texte se configure lors de l'opération même de la lecture, dans la rencontre avec un lecteur, en fonction de son attente, de ses questions et de sa curiosité. L'historien des pratiques lettrées sur la longue durée retrouve ici comme un avatar de la virtuosité narrative et poétique des aèdes qui s'adaptaient à leur auditoire et pouvaient conter différemment les mêmes histoires, selon les circonstances de l'improvisation. Dans l'"oral poetry", chaque version est autosuffisante, autonome, elle actualise une forme et un récit dans un répertoire de motifs combinables à volonté. De même, dans les bibliothèques contemporaines qui proposent les livres humains, chaque lecteur " lit " un texte différent, même lorsqu'il lit le même livre que les autres. Le livre 
humain est à la fois le medium et le message, le texte et l'auteur, et ce texte est indissolublement lié à son support, il ne peut pas être objectivé indépendamment de l'humain qui le porte. D'autre part, ce livre humain est unique, non reproductible, irremplaçable. Une transcription, un enregistrement vidéo ou audio ne conserveront qu'une infime fraction de cette textualité fluide et infinie qu'il déroule lors de chaque lecture. Avec le livre humain, le texte s'écrit par la voix, la lecture croise le regard et l'écoute. On pourrait même dire que c'est le livre lui-même qui se lit à haute voix. Il est aussi son propre auteur. En fait, le livre vivant choisit son début et sa fin, découpe ses chapitres, s'écrit lui-même, ou plutôt se réécrit sans fin en fonction du lecteur qui l'écoute. À moins que ce ne soit précisément cette écoute qui déroule le fil du récit, des confidences, et écrive une version éminemment personnelle et intime, unique et non reproductible dans ce libre espace de variance et de fluidité.

On sait que tout lecteur laisse des traces, visibles ou invisibles, sur les livres : annotations marginales, surlignements, traits et croix, signes de ponctuation du parcours de l'œil et de l'esprit. Cette sémiologie des marges et des interlignes contribue à baliser le texte, à indiquer des carrefours, des lignes de fuite ou d'errance. Le lecteur peut-il marquer, annoter et surligner un livre humain ? Il le modifie par sa lecture et son écoute, ses questions, ses réactions. Il participe au processus d'une écriture de soi, d'une écriture du moi, par laquelle le livre humain écrit sa propre histoire au moment même où il la raconte à son lecteur. Chaque livre vivant garde peut-être la mémoire des lectures antérieures qui ont contribué à la stratigraphie de ses niveaux d'écriture. Le livre humain serait-il un palimpseste?

\section{Christian Jacob}

